\title{
Marek Biszczanik
}

Universität Zielona Góra, Zielona Góra

\section{Das Erste Stadtbuch aus Schweidnitz im Lichte der Textallianzen- und Textsortenproblematik}

Neben Urkunden und Briefen gehörten Stadtbücher verschiedener Art zu den populärsten Formen der handschriftlichen Überlieferung im Spätmittelalter, und zwar schon seit dem 13. Jh. Beim Übergang zur Frühen Neuzeit spielten sie nach wie vor eine große und, man kann sagen, immer größere Rolle im öffentlichen Alltag. Im Unterschied aber zu den „nach außen“ gerichteten Schriftformen, wie Urkunden, Briefe usw., dienten die Stadtbücher (häufig auch ,Amtsbücher" genannt, obwohl diese Bezeichnung eher als Oberbegriff für ,Stadtbücher" und andere amtliche Dokumente geeignet zu sein scheint) von Aufzeichnungen unterschiedlichen Typs eher für interne Zwecke der jeweiligen Institution, sei es eine städtische Behörde oder eine fürstliche, königliche oder kaiserliche Kanzlei. Es ist dennoch relativ schwierig, nur aufgrund dieses einen Merkmals, also dieses internen Gebrauchstyps, ,Stadtbücher' ohne Weiteres als eine geschlossene und präzise umrahmte Textsorte zu definieren (vgl. Meier/Ziegler 2001: 217-220, Ziegler 2003, Fix 2008, Wolf 2009: 1691-1705). Abgesehen von diversen Zwecken der Buchführung lassen sich nämlich unter den Stadtbüchern Randfälle finden, die generell die üblichen Merkmale des ,Stadtbuches' aufweisen (vgl. Simmler 1996: 600 f.), doch aber unter einem gewissen Aspekt, formal oder inhaltlich, teilweise andersartig sind und von daher die theoretischen Rahmen der Textsorte überschreiten, die nach Brinker (2010: 120, 124) als „konventionell geltende Muster sprachlicher Kommunikation“ - hier auf der Ebene des Graphemischen - zu verstehen sind.

Nach der texttypologischen bzw. textlinguistischen Analyse von mehreren spätmittelalterlichen Stadtbüchern scheint die Ursache ihrer Heterogenität beinahe auf der Hand zu liegen. Ein typisches Stadtbuch stellt nämlich kein thematisch oder formal einheitliches Gebilde dar; es ist vielmehr eine nach gewissen 
Anordnungsprinzipien zustande gekommene „Symbiose“ von unterschiedlichen, gebrauchsfunktional eingeführten Kleintexten in einer Ganzheit, die als eine Mitüberlieferung bezeichnet werden kann (vgl. Kuhn 1969: 261-280, Schmidt-Wiegand 1992: 69-86). Ausnahmefälle gibt es zwar auch; dies ist vor allem in den größten Kanzleien der Fall, in denen wegen der Spezifizierungsmöglichkeit bzw. -notwendigkeit einzelne Bücher für unterschiedliche Bereiche des städtischen Lebens geführt wurden. Es ist vielmehr eine Ansammlung von Aufzeichnungen diverser Art (von Repräsentanten verschiedener Textsorten), in der neben kalligraphisch ausgestalteten Textpassagen und historisch gewichtigen Akten knappe oder flüchtig gekritzelte alltägliche Notizen städtischer Schreiber bezüglich ganz trivialer Ereignisse aus dem Privatleben der Stadtbürger ihren Platz fanden. Diese Vielartigkeit von in einem Buch gesammelten Dokumenten stieg übrigens mit der Zeit, was mit der fortschreitenden Spezifizierung und Professionalisierung (vgl. Becker 2006: 101) einzelner Bereiche des Kanzleiwesens verbunden war. Somit wären ,Stadtbücher' im texttypologischen Sinne nur theoretisch und ganz pauschal als eine Textsorte zu bezeichnen; als tatsächlich vorhandene historisch überlieferte Textkomplexe sollen viele von ihnen vielmehr mit der Bezeichnung, Textallianz - „Summierung verschiedener Textarten“ (Greule 2012a: 82) - versehen und als solche analysiert werden. Im Weiteren wird eben versucht, diese Annahme an einem ausgewählten Beispiel zu erörtern, wobei unter der Bezeichnung, Textallianz ' das Miteinandervorkommen von diversen Texten, die gegebenenfalls auch unterschiedlichen Textsorten angehören können, verstanden wird.

Nicht seit gestern versucht die Textlinguistik die gegenwärtigen sowie die historisch überlieferten Texte bestimmten Klassen oder Typen zuzuordnen. Diese mehr oder weniger gelungenen Klassifizierungsversuche erstrecken sich im Großen und Ganzen in zwei Richtungen. Zum einen ist man bemüht, Dachbegriffe für Textmengen zu finden, die sich durch bestimmte gemeinsame oder zumindest ähnliche Charakteristika auszeichnen. Auf diesem Weg gelangte man schon längst zum Textsortenbegriff, in dessen Rahmen Textexemplare, die bestimmten Textmustern entsprechen sollen, gewisse, relativ präzise angelegte Voraussetzungen, aber auch praktische kommunikative Aufgaben zu erfüllen haben (vgl. Adamzik 2000: 91-112). Darüber hinaus stellte man bereits fest, dass sich die Textmengen im inhaltlichen bzw. formalen Aspekt zu quantitativ so gut wie unbeschränkten Diskursen anhäufen lassen. Im Zuge textlinguistischer Forschung sowie im Rahmen der sog. Intertextualität (vgl. Adamzik 2004, Fix 2008: 31 ff.) richtete man den Blick auch auf die innere Konstitution der Texte als Ganzheiten bzw. auf die der Textzusammensetzungen. So erwies es sich als angebracht, eine in der Sprachwissenschaft relativ innovative textlinguistische Qualität einzuführen, nämlich die der ,Textallianzen“. Die Reichweite dieses Begriffes liegt den eigentlichen exemplarischen Textexemplaren viel näher als die der Diskurse, die ja viel umfangreichere Textkorpora und dazu von einer Weitwinkelperspektive umrahmen. 
In vorliegender Studie wird auf den vielschichtigen und relativ umfangreichen Text des Ersten Stadtbuches der niederschlesischen Stadt Schweidnitz fokussiert, das zurzeit im Breslauer Archiv aufbewahrt wird (Archivsignatur 180 im Bestand der Bücher der Stadt Schweidnitz). Die Handschrift liegt in Form eines relativ dicken Buches (129 Seiten) vor. Das Korpus bereitet keine Schwierigkeiten, was seinen Entstehungsraum angeht. Alle einzelnen Textteile, aus denen das Buch besteht, wurden in Schweidnitz niedergeschrieben, und zwar im Rahmen der Tätigkeit von Kanzlisten des dortigen Stadtrates. Auf den ersten Blick scheint auch die Zeit der Fixierung gar nicht problematisch zu sein. Die archivarische Datierung gibt ganz ausdrücklich die Anfangs- und Enddaten als 1321-1330 an. Nach der genaueren Lektüre des ganzen Buches findet man aber hier und da dazugeschriebene Einträge aus späterer Zeit $(1344,1375)$, die aber ohne besondere Hervorhebung in den Wortlaut des Ganzen inhaltlich und texteditorisch integriert wurden und als solche nicht auffällig sind. Dies alleine - also die Ausdehnung der Entstehungszeiten von einzelnen Textteilen ${ }^{1}$ - wäre schon ein gewisses Indiz dafür, dass der Textvorrat des Buches insgesamt als eine Textallianz angesehen werden darf. $\mathrm{Zu}$ ähnlichen Schlüssen kam in seiner Studie zu einem der Stadtbüchern aus Krakau Józef Wiktorowicz (2011: 152), der behauptete, die „Textallianzen zwischen den einzelnen Textsorten, die im Stadtbuch enthalten sind, ergeben sich hauptsächlich aus der Tatsache, dass der Stadtschreiber die unterschiedlichen Textsorten in Form von Protokollen festhält. Die sprachliche Form weist deshalb viele Ähnlichkeiten auf, während ihre Funktion in der Stadtgemeinschaft recht verschieden sein kann“".

Das Hauptanliegen der Studie ist die Darstellung des den breiteren Kreisen der Linguisten ganz wenig oder gar nicht bekannten handschriftlichen, archivarischen Sprachmaterials, der Methode seiner Erforschung und der textologischen Untersuchung seines Inhalts, und zwar auf die schon in führenden Abhandlungen zum Textallianz-Charakter der Stadtbücher bewährte Art und Weise (vgl. Waligóra 2009, Wiktorowicz 2007: 275 ff., 2011: 139 ff.). Durch die Erörterung der Textbelege, die im vorliegenden Beitrag präsentiert werden, soll nämlich nachgewiesen werden, dass einzelne Eintragungen in den niederschlesischen Stadtbüchern häufig über die Grenzen eines in sich geschlossenen Protokolls hinausgehen und es bedürfen, als Textallianzen (verstanden als „Konglomerate unterschiedlicher Texte“, die auch diversen Textsorten angehören können; vgl. Meier/Ziegler 2001: 222) präziser definiert zu werden. Aus editorischen Gründen konnten hier nur ausgewählte Beispiele einzelner Textbausteine gezeigt werden (vgl. Simmler 1996); jedem folgt jeweils eine buchstabengetreue Transliteration sowie ein Versuch, auf die Funktion des gegebenen Textbausteines in der Textallianz und auf seine Relation zu den sonstigen Textallianzteilen sowie der historischen Textsorte hinzuweisen (vgl. Simmler 2007: 452).

${ }^{1}$ Messina (2013: 53-92) verwendet hierfür auch den Begriff der „(makro- und mikrostrukturellen) Textbausteine“. 
Zunächst kommen wir zum methodischen Problem der Definierung dieses immer noch jungen Terminus. In Frage kommen generell 2 Deutungsansätze oder -tendenzen der , Textallianz'. Anzufangen wäre mit Simmlers Konzept von „Gruppierungen von Textexemplaren“, das jedoch eigentlich mehr Inhalt umfasst, als dieses knappe Stichwort suggeriert. Da handelt es sich nämlich zum einen um das makrostrukturelle und sozusagen nach innen gerichtete Verhältnis eines Textstückes als einer geschlossenen Spracheinheit zu dessen einzelnen Teilen; zum anderen blickte das Simmlersche Konzept von der Perspektive eines Textexemplars nach außen und ließ dessen Platzierung im Rahmen viel umfangreicherer Textmengen beobachten und bewerten (vgl. Simmler 1996). Schwarz (2001: 10) erinnerte allerdings an die ursprüngliche Mehrdeutigkeit des Begriffes. Über einzelne Autoren, die den Begriff der Textallianz gebrauchten, und über ziemlich auseinandergehende Deutungsweisen und dementsprechend Anwendungsfelder des Terminus könnte eine getrennte Abhandlung geschrieben werden. Hier muss es ausreichen, wenn wir insgesamt fünf grob abgezeichnete Gebrauchsweisen des Begriffes anführen, ohne sich in Einzelheiten einzelner Ansätze zu vertiefen. So könnte als ,Textallianz' eine Gesamtheit von überlieferten Repräsentanten mehrerer Textsorten im Rahmen einer Dokumentform (beispielsweise eines Buches) verstanden werden. Etwa dasselbe könnte sich aber auch auf noch umfangreichere Rahmen ausdehnen und die traditionellen Grenzen eines Dokuments oder gar eines Buches überschreiten. Diese Ausdehnung kann sogar beinahe extrem weitergeführt werden, und zwar zu einer theoretisch quantitativ unbeschränkten Menge von Textstücken, die sich allerdings auf eine gewisse Weise auf einen „textsortenartigen“ Nenner zurückbringen lassen. Der Begriff, Textallianz" wird jedoch auch ganz abstrakt verstanden und verwendet, und zwar nicht als eine Textform unterschiedlichen Umfangs und diverser Struktur, sondern als eine inhaltliche Relation zwischen einzelnen Textstücken oder eines Textexemplars zu einem größeren Textbestand. Schwarz (2001: 11) postulierte zusammenfassend eine übergreifende Akzeptanz für alle bisherigen Auffassungen des Begriffes und äußerte den Wunsch gar ,nichts davon auszuschließen“. Einerseits sind nämlich die Abgrenzungen zwischen den Deutungsversuchen verschwommen, andererseits geht es in ihnen allen letztendlich sowieso um Konnexe von Texten unterschiedlicher Textarten. Umfang, Angehensweise oder größere oder kleinere Bezugnahme auf die Textsortenproblematik spielen hier nur eine zusätzliche und präzisierende Rolle.

Das hier als Basiskorpus ausgewählte Erste Stadtbuch aus Schweidnitz (1321-1330) gibt die Möglichkeit, sowohl seinen Inhalt in toto, also als eine Textallianz, als auch die innere Struktur seiner einzelnen Textstücke, als Repräsentanten einzelner Textsorten, zu untersuchen. Was aus einem Textkomplex eine Textallianz macht, ist vor allem die Verflechtung von zwei bzw. mehreren Textsortenrepräsentanten in einem geschlossenen Rahmen - sei es ein umfassendes Dokument oder ein ganzes Buch sensu stricto. Unter dem Begriff, Text- 
sorte wird hier allerdings ein funktional spezifizierter Texttyp verstanden (vgl. Heinemann 2001b: 507-523). Denn „Texttypen sind etablierte Lösungsmuster für wiederkehrende textuelle Aufgaben, [...] mehr oder weniger stark verfestigte Traditionen des sprachlichen Handelns mit Texten“ (Gloning 2010: 174). Beide Voraussetzungen - die Anhäufung von typologisch heterogenen Textstücken in einem Buch und die strukturelle Konstellation von texttypologisch diversen Textpassagen in einem einzelnen Dokument des Buches - lassen sich im Schweidnitzer Ersten Stadtbuch nachweisen. Selbstverständlich sind die Grenzen zwischen einzelnen Textsorten oftmals verschwommen; die Anordnung von Dokumenten oder von deren Teilen zu den einzelnen Textsorten kann hier also, wie auch sonst in vielen historischen Gebrauchstextsammlungen, problematisch sein (vgl. Heinemann 2001a: 523-546). Alles in allem lässt sich aber der Inhalt des Buches der sehr breit angesetzten und vielschichtigen Auslegung des Textallianz-Begriffes von A. Schwarz (2001: 11) unterordnen, die im allgemeinen Umriss oben erläutert wurde.

Texttypologisch repräsentiert die Korpustextsammlung ein Konglomerat der Repräsentanten von etwa drei verschiedenen Textsorten ${ }^{2}$ (lateinische Textpassagen haben hier nur einen Randcharakter), die jeweils bestimmte syntaktische Muster zur Realisierung von den einzelnen kommunikativen Aufgaben aufweisen (vgl. Ehlich 1994: 18-41, Ziegler 2005: 271 ff., Gloning 2010: 181), wobei eine von ihnen stets dominiert und von den sonstigen ergänzt wird. Der Haupttyp des Textes im Stadtbuch kann grob als ein ,Protokoll des Stadtrates` bezeichnet werden. Darunter verstehen wir jegliche, vom Stadtrat verfertigte Aufzeichnungen, die die Angelegenheiten der Stadtbürger, -institutionen bzw. -behörden betreffen, die während der Ratssitzungen ausgetragen wurden. Inhaltlich beziehen sie sich auf ein sehr breites thematisches Spektrum, daher ist diese Dokumentengruppe qualitativ und quantitativ sehr inhomogen. ${ }^{3}$ Was aber alle diese Notizen inhaltlich verbindet, ist eben der Bezug auf Ereignisse, die entweder direkt in Schweidnitz (mit der nächsten Umgebung) stattfanden oder für die Stadt und ihre Einwohner vom Belang waren. Unten wird eine Textpassage des Korpustextes (mit der Transliteration) präsentiert (Abb. 1; Blatt 17), wo Ratsnotizen zu sehen sind, die zum oben erwähnten Haupttextbaustein des Buches gehören:

${ }^{2}$ Dass die Grenzen zwischen einzelnen, historisch etablierten Textsorten oftmals nicht scharf festlegbar sind, hat man auch in anderen Studien festgestellt. So ist auch eine präzise und zweifelsfreie Zuordnung eines historischen Textstückes (vor allem eines Fragmentes einer größeren Texteinheit, wie hier - eines Stadtbuches) nicht immer ohne Weiteres möglich. In diesem Kontext sprechen z.B. Meier u. Ziegler sogar von ,Übergangsklassen“ zwischen einzelnen „,musterhaften“ Idealtypen von Textsorten (vgl. Meier/Ziegler 2004: 149).

${ }^{3}$ Neben ausführlich dargestellten Angelegenheiten allgemeiner Geltung, die die ganze Stadtgemeinschaft betreffen konnten, besprach man in den niederschlesischen Stadtbüchern in ganz kurzen Einträgen solch alltägliche Ereignisse, wie Diebstähle von Zuchttieren, Rechte auf von Bäumen gefallenes Obst u.ä. 


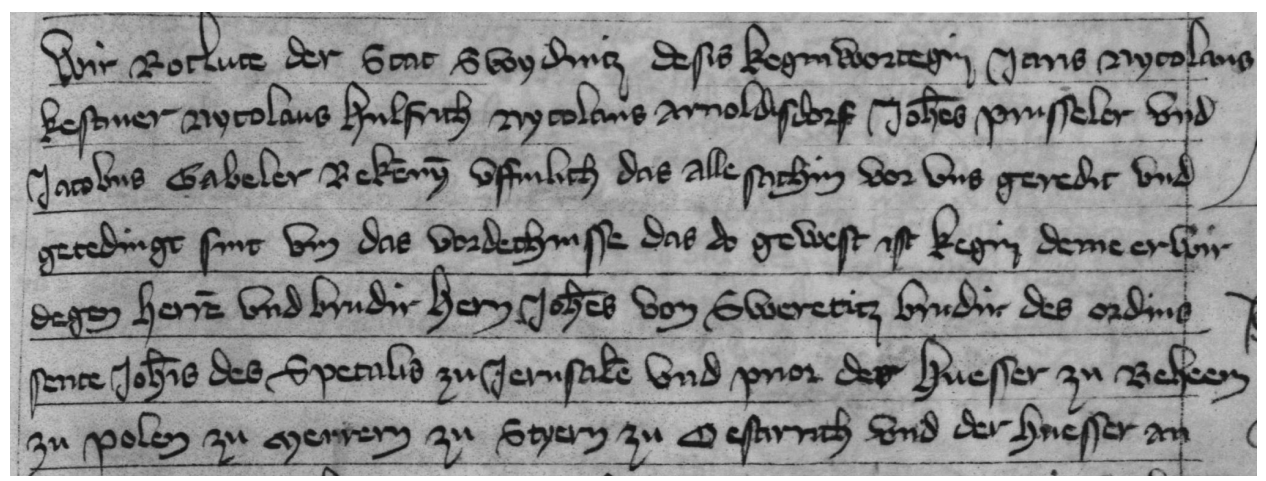

Abb. 1: Die ersten 7 Zeilen vom Blatt 17 des Korpustextes

\section{Zum Inhalt dieses Fragmentes: [Blatt 174:]}

Wir Rotlute der Stat Swydnicz desis keginwortegin Jaris Nycolaus kestmer Nycolaus ulfrich Arnoldisdorf Joh(ann)es prusseler vnd Jacobus Gabeler Beke(n)ny(n) vffinlich das alle sachin vor vns geredit vnd getedingt sint vm das vordechnisse das do gewest ist kegin deme erwirdegen herre(n) vnd brudir hern Joh(ann)es von Swereticz brudir des ordins sente Joh(ann)is des Spetalis zu Jerusale (m) vnd prior der huesser zu Beheem zu Polen zu Merrern zu Styern zu Oestirrich vnd der huesser andirswo wo die gelegen sint des selbin ordins von sinent wegin vnd von sines ordins wegin vnd von aller siner vru(n)de wegin alz an eyme teile / vnd von her frantzke(n) Slu $e^{s} \beta$ vnd Petir keyser di do angeborn Brudir sint gewest hern Joh(ann)is keysers selgisgedechnisse der eyn brudir ist gewest des obgn(anten) ordins vnd von zachinkirchin vnd von alle(n) andirn erre vru(n)de wegin / vm vnd von des obgn(anten) hern Joh(ann)is keysers wegin alz von deme andirn teile / alzo vorrichtit ist / vnd vorsunyt vru(n)tlich vnd gutlichin an alle argelist kegin allen $p(e r)$ sone (n) sie sint geistlich adir werlich / an beidin teilen / alzo das ma(n) der selbin sachin ny(m)myr mer gedencke(n) sal mit wurten nah mit werein / an beide(n) siten kegin allen den di do nv lebin adir hernacher zuku(n)ftig werdin / Ouch hat der obgn(ante) her Joh(ann)es Prior des obgn(anten) ordins angeseen got vnd meru(n)ge des gotis dinst daz man stete sal meren / wo kan vnd mag gote vnd siner Mutir zo loebe vnd zu eren vnd des obgn(anten) herre(n) / hern Joh(ann)is keys(irs) Brudir vnd vru(n)de demutege bete vnd erre(n) willegin dinst den sie deme ordin vffte vnd vil irczegit han. vnd hernacher irczegin werdin in zu(n)ktigin czitin vnd hat enphangin vnd gegebin durch d(en) got vnd durch des obgn(anten) hern Joh(ann)is keysers vru(n)de demutege bete wille tzween prioren den ordin alzo Joh(ann)is Beyirn des obgn(anten) hern Joh(ann)is keysers Swestir soin wen der gewiet wirt zu eyme subdiacon zo sal her werdin gekleidit mit des obgn(anten) ordins kleyt /

\footnotetext{
${ }^{4}$ Hier und im Folgenden handelt es sich um die originale Seitennummerierung.
} 
vnd die aindir Petrus de amore we(n)ne der gewiet wirt zu Prister der ouch eyn vru(n)d gewest ist des obgn(anten) hern Joh(ann)is keysers so sal ouch gekleydit werdin mit des obgn(anten) ordins kleyd / nach des ordins gewonheit / do bie sint gewest / die erbern herre(n) her Bernhard von Rothewicz her han(os) von der Cirnow Ritter vnd Bartusch von Botschaw vnd / Paulus vnß(er) Statschriber deme wir d(er) sachin beuolin zuschribin.

In dem Text kann man die Verflechtung von Fragmenten zweier für Stadtbücher charakteristischer Eintragungstypen ${ }^{5}$ beobachten. Die erste Gruppe von Eintragungen kann als spätmittelalterliche öffentliche Korrespondenz bezeichnet werden; der andere Typ wäre eine breit verstandene Rechtsweisung. Die Makrostruktur des Dokuments wird so konstruiert, dass Elemente der Briefgestaltung die rechtsspruchartigen Formeln umklammern. Im zitierten Protokoll verläuft die Verzahnung von den Repräsentanten der beiden Texttypen ${ }^{6}$ etwa wie folgt: Angefangen wird traditionell mit kurzer intitulatio, in der die Ratsherren von Schweidnitz sich selbst namentlich (was damals übrigens relativ selten war) und als Absender vorstellen (Für dieses Textstück ist die Verwendung der 1. Pers. charakteristisch.). In diesem einführenden Teil platzierten die Ratsherren auch einen Ersatz für die fehlende Datierung, und zwar in Form des Vermerkes: desis keginwortegin Jaris. Im Falle einer losen Urkunde könnte es die richtige datatio natürlich nicht ersetzen; das präsentierte Dokument ist jedoch ein Baustein des ganzen Buches, in dem die einzelnen Jahrgänge mehrmals angegeben werden. Noch knapper als die Absendertitulatur ist die den Willen der Aussteller präsentierende promulgatio. Für den Rechtsweisungs-Teil ist die Verwendung der Vergangenheitsformen für die von den Ratsherren vorgefundene Sachlage sowie der Periphrase mit dem Auxiliar sollen, und zwar an den Stellen, an denen auf weitere Schritte der Beteiligten hingewiesen wurde, typisch.

Für spätmittelalterliche Kanzleitexte ist es charakteristisch, dass einzelne Sätze sich nicht selten über ganze Dokumente oder zumindest über größere Textpassagen erstrecken und als Nebensatzkomplexe (manchmal mit hauptsatzlosen, sehr umfangreichen Nebensätzen) angesehen werden können (vgl. Waligóra 2009: 18 20). Dies bemerkte auch Simmler (2001: 140), dessen Ansatz der syntaktischen Gliederung eines spätmittelalterlichen Dokuments auf der Markierung von textinneren Inhaltsteilungskennzeichen beruht und Teilsätze als Elementarsätze ansehen lässt. Die Folgen einer solchen Textstrukturierung sehen wir auch im untersuchten

${ }^{5}$ Von „Eintragungen“ und „Gruppen von Eintragungen im Stadtbuch“ schreibt auch Meier (2004: 116).

${ }^{6}$ Den „Texttyp-Begriff [...], der Teiltexte oder Sequenzen charakterisiert“, verwenden u.a. Engberg/Nølke (1997: 169). „Ein Texttyp ist eine besondere Art, Text [...] zu präsentieren. Beispiele von Texttypen sind etwa der argumentative, der deskriptive und der explikative Texttyp. [...]“ (ibid.). 
Textexemplar. Die intitulatio (mit Datierungsangabe) und die promulgatio machen den Hauptsatz aus. Danach beginnt der lange und mehrere detaillierte Informationen beinhaltende Nebensatz bzw. Nebensatzkomplex.

An dieser Stelle setzt der andere Eintragungstyp ein: die Verfügung, die nach Busse (2000: 671) als eine der Textsorten der Rechtsprechung zu begreifen ist. Gerade im Nebensatzkomplex wird der eigentliche Inhalt der Sache ausgelegt. Zuerst wurde die narratio ausgeführt, wo die Aussteller die Umstände und bisherige Festlegungen der untersuchten Sache darstellten. Daraus resultieren weitere Bestimmungen des Stadtrates, nach denen die rechtliche Weisung in Form einer dispositio mit einzelnen Klauseln vorrichtit ist. In diesem Teil lassen sich schon wieder einzelne Hauptsätze aussondern, und zwar vor allem da, wo Dispositionen des Stadtrates mit Hilfe des Modalverbs sollen, dessen finite Formen zum Kern des Satzes werden, zum Ausdruck gebracht werden. Somit lässt sich hier also eine gewisse pragmatisch-syntaktische Relation bemerken: Die eigenen Faktenangaben des Stadtrates wurden generell in den Hauptsätzen formuliert; andere, sozusagen vorbereitende Informationen, finden sich meist in den Nebensätzen bzw. Nebensatzkomplexen. Kurz vor dem Ende des Aktes endet auch sein „rechtlicher Teil". Den Abschluss des Dokuments bildet nämlich eine kurze testatio, in der die Zeugen des Rechtsaktes genannt wurden. Dieses abschließende Element ist ein traditioneller Bestandteil aller mittelalterlichen bzw. frühneuzeitlichen Beschlüsse von Behörden des städtischen Rechts. Somit vereint dieses Element Voraussetzungen beider Textsortengruppen.

Die einzelnen Bucheinträge unterscheiden sich nicht nur formal, sondern auch im Sinne ihrer objektiv gemeinten „Gewichtigkeit“. Das oben dargestellte Dokument schildert Bestimmungen des Schweidnitzer Rates bezüglich der Institution, deren Wirkungsfeld sich weit über die Mauern der Stadt erstreckte und etliche Menschengruppen betraf. Der Prototyp des ,Protokolls des Stadtrates', wie es Greule (2012b: 285) nannte, kann aber auch die Schilderung von Dingen viel geringerer Geltung umfassen. Diese Geltungsabstufung wirkt sich auch auf den Kompliziertheitsgrad der Makrostruktur der Urkunde auf. So finden wir im Buch Dutzende von Einträgen, in denen Bestimmungen verewigt wurden, deren Geltungsbereiche sich auf eine bzw. zwei Personen erstreckten. Und dies erkennt man schon an der Oberflächenstruktur des Textes selbst: er ist viel knapper, und zwar nicht nur wegen der (auch rechtlichen) Unkompliziertheit des besprochenen Falls, sondern auch wegen der Auslassung von fakultativen Textmusterteilen. Alles, was nicht in Direktbeziehung zur nötigen und kurzen Narration des Stadtrates stand, wurde ausgespart. Selbst die Disposition, also der Kern des Aktes, ist in der narratio versteckt. Sie resultiert einfach aus der Beschreibung der Sache und schmilzt mit narrativen Angaben in einer knappen und präzisen publicatio zusammen (vgl. Rachoinig 2009: 105). Denn ,nicht in jedem Fall ist das prototypische Muster vollständig realisiert“ (Greule 2012b: 285). Selbstverständlich ist in jedem typischen Stadtbuch eine ganze 


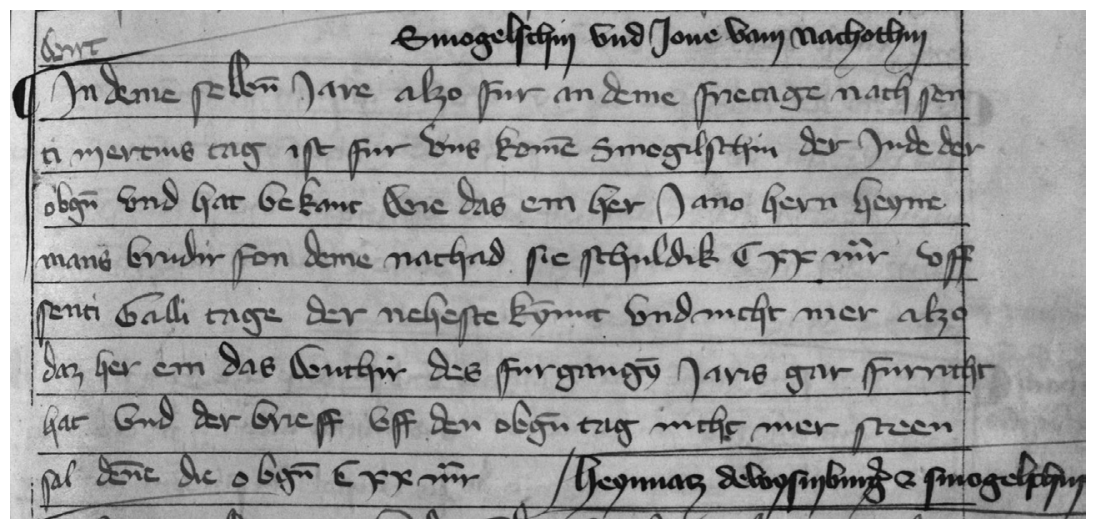

Abb. 2: Das Blatt 22 des Korpustextes (ein Fragment)

Menge von solchen Einträgen zu finden, in denen Textteile fehlen, die aus logischen Gründen ausgelassen werden konnten. Hier nur ein Beispiel (Abb. 2; Blatt 22):

Die Transliteration der Handschrift:

\section{Smogelschin vnd Jone vam Nachothin}

Jn deme selb[i]n Jare alzo fur an deme frietage nach senti mertins tag ist fur vns kome[n] Smogilschin der Jude der obgn[ante] vnd hat bekant wie das em her Jano hern heynemans brudir fon deme nachad sie schuldik CXX m[a]r[k] vff senti Galli tage der neheste ky[m]mit vnd nicht mer alzo daz her em das wuchir des furgangy [n] Jaris gar furricht hat vnd der brieff vff den obgn[anten] tag nicht mer steen sal de[n]ne die obgn[anten] CXX m[a]r[k]

Am Anfang des Eintrags sehen wir eine etwas reduzierte datatio, in der sich die Jahresangabe auf oben eingetragene Einschreibungen stützt, in denen die Datierung mindestens einmal vollständig angegeben wurde. Die datatio geht in die Schilderung der Geschehnisse, die sich an dem angeführten Tag ereigneten, wobei die Finalität der Sachlage periphrastisch (dabei wurden nur durative Verben mit dem Perfektivitätspräfix ge- versehen) und die Verordnung des Stadtrates - mit dem futurisch gekennzeichneten Auxiliar sollen wiedergegeben wurde.

Der oben präsentierte Texttypus stellt mit seinen Repräsentanten, wie angedeutet, den Kern der Dokumente der Korpusquelle dar. Daneben begegnen wir nicht selten dem Auftreten von Belegen anderer Textsorten. Was diese einzelnen Textstücke voneinander genug different macht, dass es von Vertretungen differenter Textsorten, die sich übrigens alle noch weiter untergliedern ließen, die Rede sein darf, ist in erster Linie ihr Inhalt und ihre Struktur. Ein zusätzliches Indiz wäre die Schriftart und die Schreibweise, die beim Notieren einzelner Dokumententypen von den Kanzlisten gebraucht wurden. Dies wird sich ebenfalls in den unten angebrachten Beispielen (siehe die Abbildungen zum Beitrag) sehen lassen. 
Aus der Textsorte der sogenannten Protokolle des Stadtrates müssen Akten ausgeklammert werden, die sich nicht auf dessen Petenten beziehen, sondern in die Zuständigkeit der Vertreter des Rates selbst fällt. Im Buch finden wir Einträge, die texttypologisch als interne Bestimmungen des Stadtrates bezeichnet werden können. Somit stellen sie hier eine separate Einzeltextsorte im Rahmen der Textallianz ,Stadtbuch' dar. Unten (Abb. 3; Blatt 15) ein entsprechendes Beispiel dafür:

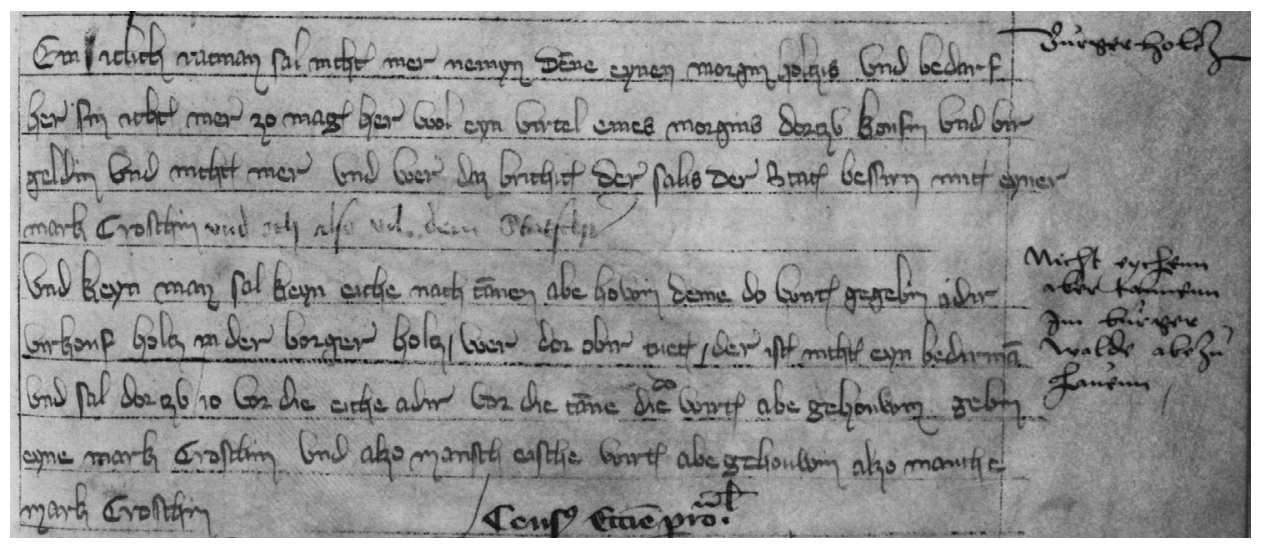

Abb. 3: Blatt 15 des Korpustextes (ein Fragment)

\section{Zum Inhalt dieses Textstücks:}

[am Rande: Burgerholcz] Ein iclich ratman sal nicht mer nemyn de(n)ne eynen morgin holczis vnd bedarf her sin icht mer zo mag her wol eyn virtel eines morgins dorczu koufin vnd virgeldin vnd nicht mer vnd wer daz brichit der salis der Stat bessirn mitt eyner mark Croschin [...] vnd keyn man sal keyn eiche nach ta(n)nen abe howin deme do wirt gegebin adir virkouf holcz in der borger holcz / wer dor obir tuet / der ist nicht eyn bedirma(n) vnd sal dor czv io vor die eiche adir vor die ta(n)ne die do wirt abe gehouwin gebin eyne mark Croschin vnd alzo mansch eische wirt abe gehouwin alzo manche mark Croschin [am Rande: Nicht eychenn aber tannenn Jm burger walde abezuhauenn]

In diesem Texttyp ist die innere Textstruktur relativ simpel. Den ganzen Inhalt umfassen dispositive Weisungen des Rates hinsichtlich eines bestimmten Aspektes der Berechtigungen seiner Mitglieder, die mit sollen-Periphrasen ausgedrückt werden. Die Notiz wird am rechten Rand mit einer thematisierenden Überschrift versehen. Auch dort befindet sich eine kleine Korrektur der Weisung.

Im Korpusband wurden neben solchen eher kleinstädtischen Angelegenheiten, wie die oben geschilderten, auch Anlässe allgemeinerer Geltung verewigt. Textlinguistisch gesehen haben wir es dabei mit einer separaten Textsorte zu tun. Diese könnte man als Bestätigung der Stadtrechte (bzw. -privilegien u.ä.) bezeichnen. Der jeweilige Schreiber verzeichnete jene Akten der herzoglichen Gnade generell von 
der Position des vielköpfigen Stadtrates, aber bald wird die „Narration“ in die Singularform versetzt und der Herrschende wird zu Wort gelassen. Selbstverständlich werden auf diese Art und Weise nur seine Worte wiederholt, sein Wille geäußert, was entweder einer anderen, älteren Urkunde entnommen oder nur im Namen des Herrschers niedergeschrieben wurde. Nichtsdestoweniger stellt auch dieser Texttyp ein gewisses Stil- und Formkonglomerat dar. Unten lesen wir ein beispielhaftes Fragment dieser Art - auf den 24. Juli 1328 datiert (Abb. 4; Blatt 25):

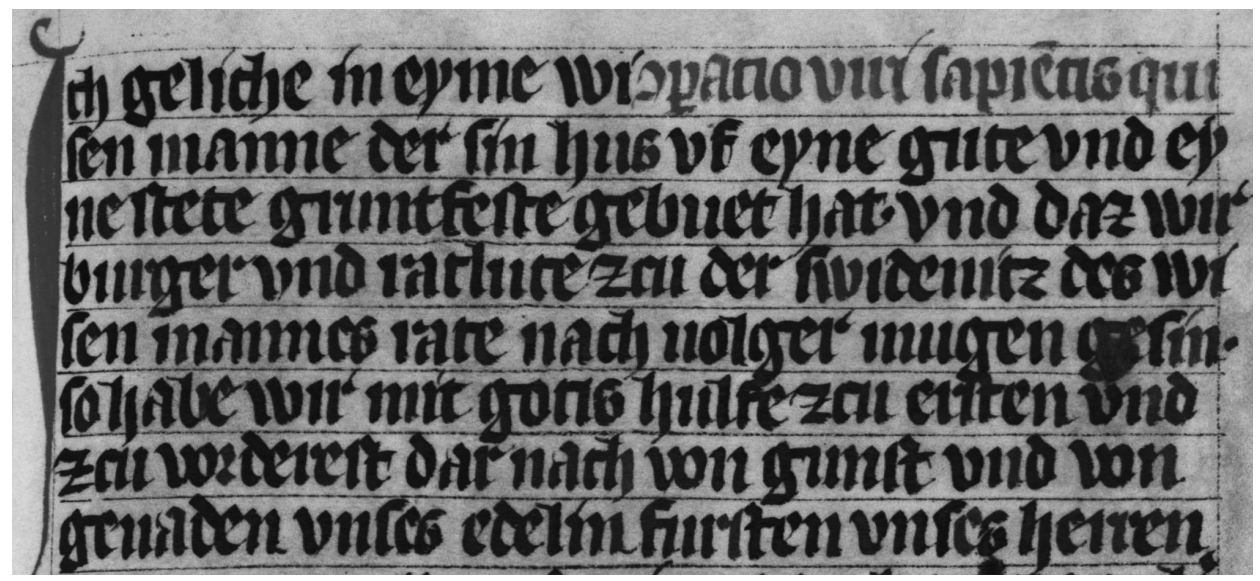

Abb. 4: Das Blatt 25 des Korpustextes (ein Fragment)

Zum Inhalt dieser Seite:

Jch geliche in eyme wisen manne der sin hus vf eyne gute vnd eyne stete gruntfeste gebuet hat vnd daz wir burger vnd ratlute zcu der swidenitz des wizen mannes rate nach uolger mugen gesin. [...]

Nach der mittelalterlichen Tradition stellten die Autoren des Textes zunächst den Bezug zu einer christlichen Weisheit her. Hier machten sie gerade aus den evangelischen Worten Matthäus' $(7,24)$ Gebrauch und paraphrasierten die Parabel vom weisen Mann, der sein Haus nur auf festem Felsen und nicht auf Sand zu bauen habe. Diese „feste Grundlage“ - diesmal für die Existenz der Stadt - sollten nämlich die vom Herzog bestätigten Rechte sein:

[...] so habe wir mit gotis hulfe zcu ersten vnd zcu widerest dar nach von gunst vnd von genaden vnses edelin fursten vnses herren herczogen polken des iungen. Herzcogen b[er]nhartz sim alle recht vnd gesetzce der stat dye wir bye syme vatir. vnd bye syme eldir vatir herzcogen polken dem alden haben gehat vnd vor ligen sin der stat die wir ouch bie sinen zciten vnd mit syme willen vnd geheyzen in andir stete zcu rechte haben gegeben zcu einer gantzcen stetikeit haben bracht. also daz die selben recht vnd die geseczce die wir von aldere be 
tracht vnd bescriben haben mit der eldesten rat von der stat vnd die wir noch bedenken vnd betrachten kvnnen vnd mvgen dye vnseren herren dem hertzcogen herlichen syn vnd der stat nuczeclich vnd vrumelich sint $\mid$ Dye selben recht hat ouch vnse herre der herzcoge mit syner getruwen manne [Blatt 25v] rat zcu eyme ewigen gedechniz bestetiget mit sinen brifen vnd mit sinen hantvesten. dye man horen sal her nach von worten zcu worten. Die hantveste vnd brif sprichit also [...]

Hier endet diese Selbsterklärung der Ratsherren (traditionellerweise in der 1. Pers. Pl. formuliert), die z.T. einen Arenga-Charakter aufweist. Es ist für mittelalterliche und frühneuzeitliche Stadturkunden typisch, dass man die Ausstellung von Akten unterschiedlicher Art mit einer kürzeren oder ausführlicheren memoria-oblivio versah, und zwar auch oft, wenn dies für das Verständnis des Ausstellungsgrundes gar nicht unentbehrlich war. Der Text geht aber nicht glatt in die Beschreibung vom Inhalt der ursprünglichen Urkunde von Bolko ${ }^{7}$ über, sondern die beiden Textteile - d.h. die Schilderung des Privilegs durch den Stadtrat einerseits und die eigentliche, aber wesentlich ältere Handfeste (in Form einer Abschrift) andererseits - werden durch eine Zwischenüberschrift verbunden (Blatt 25v): Dize hantfesti hat herczoge polke der iu(n)ge gegebin den burgern. Von dieser Stelle an lesen wir schon die Abschrift des Privilegs vom Herzog Bolko selbst. Traditionell eröffnet die Textkomposition ein kurzer invokativer Teil (Jn gotis namen amen). Danach wurde die indirekte Ursache der Urkundenausfertigung in Form einer Arenga formuliert: Daz dye genediclichen gabe di von mildir gutheit der vursten kvmen in eine vorgessinheit icht kvmen noch vallen. So ist iz nuczce vnd notdurft daz man sie eyme gedechtnis bevele vnd in eyne schrift brenge den nachkvmelingen zcu eyme bilde. Dieser Textteil fand hier seinen Platz wohl mehr der Betonung der herzoglichen Güte und Gnade zuliebe und weniger mit der Absicht, dem Leser etwas tatsächlich Informatives mitzuteilen. Der weitere Teil, der eine Zusammenschmelzung von intitulatio, promulgatio und sogar (auch wenn nur marginal) inscriptio zu sein scheint, enthüllt alles zugleich: den Namen und Rang des Urhebers des Dokuments (hier war es der Herzog Bolko von Schlesien), seinen Willen (die Aushändigung der Handfeste) sowie die Adressaten dieses Willens (die Bürger der Stadt Schweidnitz). Dieses funktional so mannigfaltige Textstück beinhaltet jedoch auch Passagen, die sich der narratio anordnen ließen; da werden ja außer den oben erwähnten, traditionsmäßig gerechtfertigten Elementen die Textausstellungsumstände genannt. Diese makrostrukturelle „Allianz" von Textfunktionsteilen lautet, wie folgt (Blatt 25v):

${ }^{7}$ Bolko II., „,der Kleine“ genannt; 1326-1368; Herzog zu Schweidnitz-Jauer (Niederschlesien). 
Darvmme wir polke von gotis genaden hertzcoge von slesie vnd herre von vurstenberc vnd zcv der swidnitz tvn kvnt an disen brife alle den die in lesen odir gesen. daz vnse getruwen burger zcu der swidnicz vor vns quamen vnd boten vns betelichen. daz wir ir recht vnd ir gesetzce irre stat di in von vnseren vor varen vnd von vnseren elderen gegebin vnd vor geligen sint. gerichten zu liben vnd zcu loben vnd zu bestetigen. vnd bestetigit dar bje zcu behalden.

Die Verdienste der Schweidnitzer Herren bzw. Stadtbürger hat der Herzog Bolko offensichtlich hoch geschätzt, weil er ihre Bitten durchaus willkommen hieß. Dabei überfließt der narrative Teil in die dispositio, in der der Willen bzw.

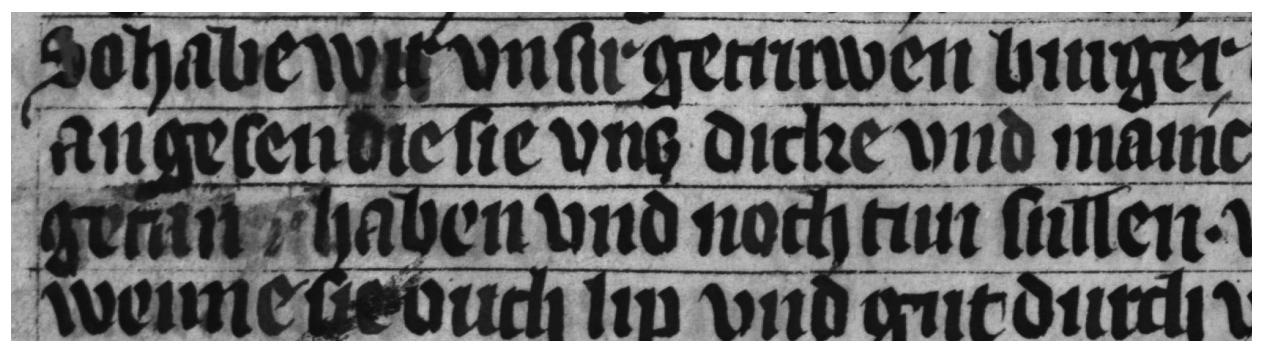

Abb. 5: Das Blatt 25v des Korpustextes (ein Fragment)

die Gunst des Herrschers Ausdruck fand, und zwar folgendermaßen (Abb. 5; Blatt $25 \mathrm{v}$ u. 26):

So habe wir vnsir getruwen burger dinst angesen die sie vns dicke vnd manicvalt getan haben vnd noch tun sullen. vnd wenne sie ouch lip vnd gut durch vnsere [Blatt 26] nicht in vurtten vz zcu setzcen vns zcu hulfe wenne wirs bedurfen. vnd wir irbete vn vorhort nicht wollen lasen. So bekenne wir offinbarlichen daz wir vnse getruwen burger zcu der swidniz bie alle den rechten vnd gesetzcen die in gegebin vnd geligen sint von vnseren elderen. vnd die sie in vnsers vetteren stete herzcogen polken vnd in andir lant gegebin haben. da wolle wir sie vnd alle ir nachkvmelinge biehalden ewiclichen vnd liben vnd loben sie vnd bistetigen in die selben recht. Dar vbir durch libe vnd sunderlich gunst die wir haben zcu vnsen vorgenanten burgeren gebe wir in vnd vorligen in ab sie icht vindin vnd irtrachten kvnnen mit iren eldisten das vns erlichen sie vnd der stat nutzcelich vnd vrumelich daz ist vnse wille vnd vnse wort.

Die herzogliche Disposition ist somit offenbart. Den Text schließt ein Komplex von traditionellen Textteilen ab, die sich meist am Ende der spätmittelalterlichen Urkunden finden lassen. Hier ist es zunächst die corroboratio, d.h. die Nennung von Mitteln, mit denen das Dokument beglaubigt werden soll: zcu enir irkunde habe wir in disen brif gegeben beuestent mit vnserem ingesigil. Folglich begegnen wir der datatio, also der Datierung und Lokation der Schriftausfertigung, die mit der ausführlichen testatio endet (Abb. 6; Blatt 26 u. 26v): 


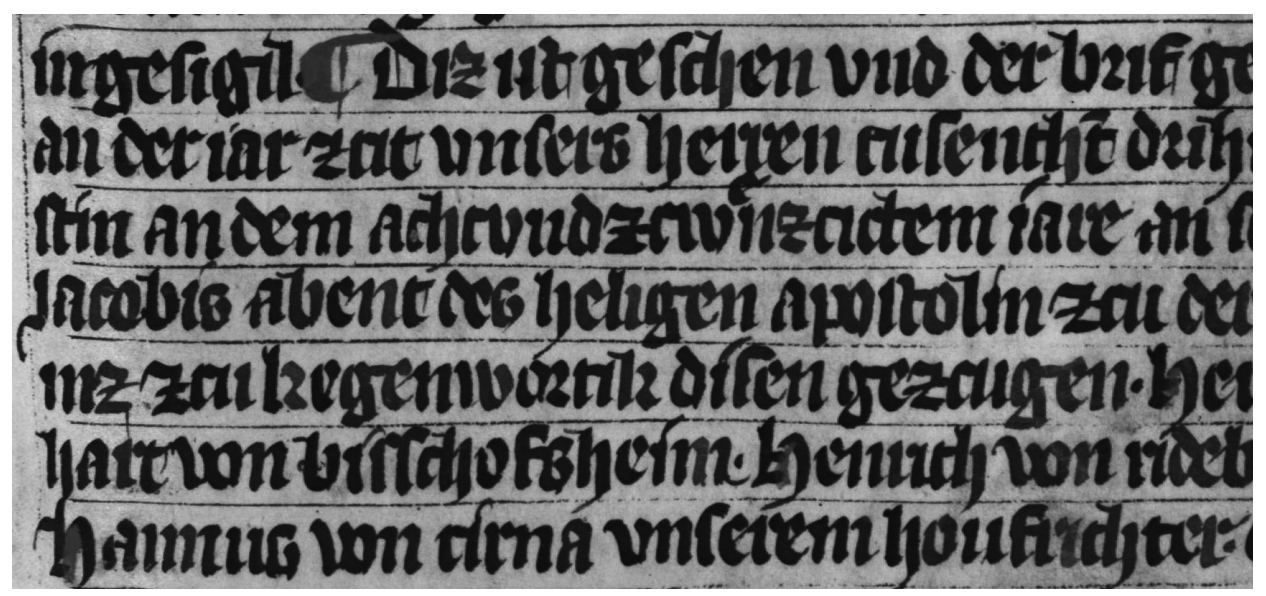

Abb. 6: Das Blatt 26 des Korpustextes (ein Fragment)

[datatio:] Diz ist geschen vnd der brif gegebin an der iar zcit vnsers herren tusentht drihundirstin an dem achtvndzcwenzcickem iare an sencte Jacobis abent des heiligen apostolin zcu der swidnitz [Zeugenbeurkundung (testatio):] zcu kegenwortik disen gezcugen. her reinhart von bisschofsheim. Henrich von rideburc Hannus von tirna vnserem houfrichter. Cunat [Blatt 26v] von betzschow. Henricus buchwalt. Sandir von der tirla. Hannus wyt von Nympz. Henricus moyes. Hernticzten vnseren lant scribere vnd anderre biderwen lute

Abschließend drängt sich eine allgemeinere Frage auf, und zwar, ob es für alle Textrepräsentanten im Rahmen einer Textallianz eine gemeinsame „Sprachform“ gibt (um in diesem Kontext nicht direkt von einer gemeinsamen „Grammatik der Intertextualität“" zu sprechen, wie es bei Greule 2012: 84 der Fall ist). Wenn man auf die Korpustexte vom traditionellen linguistischen Standpunkt schaut, dann müsste man feststellen, dass ihre ,grammatische Gestaltung“ relativ ähnlich ist. Selbstverständlich muss dabei mitberücksichtigt werden, dass in manchen von ihnen bestimmte Textteile mit den für sie typischen sprachlichen Strukturen auftreten, die dagegen bei den anderen ausgespart wurden. Demzufolge fehlt beispielsweise im Typ ,interne Bestimmung des Stadtrates' das gesamte Protokoll, also das exordium, mit allem, was es umfassen könnte, also z.B. invocatio, intitulatio oder inscriptio. Im Konzept des Dokumentes begegnen wir, wie gesagt, nur der dispositio; nicht vorhanden sind solche typischen Konzeptbestandteile, wie etwa arenga oder corroboratio. Es fehlt auch das ganze Eschatokoll mit datatio, testatio usw. Dafür aber ist die Sprache der dispositio nicht andersartig von der, die wir in den ausgebauteren Dokumenten des Buches antreffen. Mehr noch: Sie ist nicht irgendwie typisch für diese und keine andere Textsorte des kanzleilichen Frühneuhochdeutschen. 
Eine gewisse beständige und makrostrukturell charakteristische Matrix, auf Grund deren Einträge des Stadtbuches konzipiert und formuliert wurden, ist wie es sich auf dem Wege des Vergleiches einzelner Beispieleinträge (sowie weiterer, die hier nicht präsentiert werden können) erweist - die Komposition einer stets obligatorischen Disposition des Ausstellers (Urhebers) des Dokumentes und mehrerer sonstiger, aber fakultativ eingeführter Teile, von denen die meisten schon oben erwähnt wurden. Somit lässt sich der Inhalt des Stadtbuches sowohl im Ganzen als auch detailliert, d.h. anhand von Beispielen einzelner Einträge, eher als eine Textallianz als etwa eine Redekonstellation erfassen, in der das Vorkommen der Bestandteile strenger sein muss, obwohl sich die beiden Termini partiell abdecken (vgl. Steger/Deutrich/Schank/Schütz 1974, Brinker/ Antos/Heinemann/Sager 2001: 1465). Falls ein Eintrag nur aus dem dispositiven Textkern besteht, dann bedeutet es allerdings nicht automatisch, dass die Informationen, die in anderen Teilen üblich präsentiert werden, wie etwa Zeit- und Ortsangaben, Zeugennamen, weitere Umstände der Textausfertigung usw., dem potenziellen Leser in diesem Falle überhaupt nicht zugänglich sind (also etwa absichtlich ausgespart worden wären). Nicht zu vergessen ist nämlich der lineare Charakter der spätmittelalterlichen Stadtbücher, in denen die Einträge zwar oft thematisch und strukturell vielartig sind, sich aber doch meist in die chronologische Reihe der nacheinander folgenden Einzelnotizen einschreiben lassen. Dessen war man offensichtlich ganz bewusst, wenn man einzelne Einträge mit fakultativen Teilen nicht versehen hat. Alle zusätzlichen Umstände mussten in solch einem Fall entweder ohne Bedeutung sein oder sie wären in entsprechenden fakultativen Teilen der „benachbarten“ Einträge zu finden gewesen; diese systemreferenzielle Intertextualität auf der Linie Textexemplar - Textmatrix musste also auch den spätmittelalterlichen Kanzlisten intuitiv verständlich sein. Die einzelnen Protokolle als solche waren ja immer sozusagen ,visuell codiert“, sodass eventuelle „Mängel“ in ihrem Sachverhalt auch beim Leser durch Anspielung auf eine Referenzmatrix intuitiv hätten rekonstruiert werden können (vgl. Janich 2008: 189, nach Vogel 2012: 72-73).

Darüber hinaus ergibt es sich aufgrund des Korpusmaterials, dass es allen Textrepräsentanten im Rahmen des Ersten Stadtbuches aus Schweidnitz derselbe Sprachusus (samt der Grammatik, des Wortschatzes, der Syntax usw.) zugrunde liegt, wie es etwa bei den meisten sonstigen Kanzleierzeugnissen dieser Epoche und diesen Rangs in dem sprachgeographischen Raum von Niederschlesien der Fall ist. Nicht also in der Grammatik selbst sollen Kriterien für Abgrenzung und Charakterisierung einzelner Textallianzen gesucht werden. Wenn nämlich von sprachlichen, oder besser gesagt, kommunikativ-pragmatischen Strukturen die Rede ist, die umfangreicher sind als übliche und traditionell verstandene Sätze, dann muss auf das Problem des Sprach- und Textbaus auch viel breiter, sozusagen holistisch, geschaut werden. Folglich ist ein Textmuster, wie Greule (2012b: 285) mit Recht denkt, „beispielsweise das Stadtbuch, das nicht nur als heterogene Ein- 
heit verstanden werden kann“. Da geht es nämlich „um eine Kommunikationsform mit dominant dokumentativer Funktion" (ibid.).

Das Schlüsselwort ist also nicht mehr eine sprachliche (grammatische oder lexikalische) Struktur, sondern vielmehr das Potenzial eines Textes als eines Ganzen und seiner Bestandteile im Einzelnen, die ja „funktionale Textbausteine sind“ (Gloning 2010: 173), bestimmten pragmatisch zu verstehenden Funktionen (vgl. Mihm 1999: 13-37) gerecht zu werden und dabei allen anderen Textexemplaren des gegebenen Textmusters (vgl. Fix 2008: 65 ff.) stilistisch, lexikalisch und textgestalterisch bzw. textstatistisch (auch im Sinne des Type-Token-Verhältnisses; vgl. Lerchner 2002: 251, 346) ähnlich zu sein. In den einzelnen Textteilen ein und desselben Textes können ja beachtliche Unterschiede in der Wahl von Strukturen bestehen. Dennoch bildet solch ein Text eine funktional und kommunikativ effiziente Ganzheit, durch die der Schreiber sein pragmatisch angesetztes Ziel erreicht.

\section{Literatur}

Adamzik, Kirsten (2000): Was ist pragmatisch orientierte Textsortenforschung. In: Adamzik, Kirsten (Hrsg.) (2000): Textsorten. Reflexionen und Analysen. Tübingen. S. 91-112.

Adamzik, Kirsten (2004): Textlinguistik. Eine einführende Darstellung. Tübingen.

Becker, Joern-Martin (2006): Hochmittelalterlicher Professionalisierung und ihre Widerspiegelung in frühen Fachsprachen im Wendland. In: Zeitschrift für Archäologie des Mittelalters 34. S. $101-111$.

Brinker, Klaus (2010): Linguistische Textanalyse. Eine Einführung in Grundbegriffe und Methoden. 7. Aufl. Berlin.

Busse, Dietrich (2000): Textsorten des Bereichs Rechtswesen und Justiz. In: Brinker, Klaus / Antos, Gerd / Heinemann, Wolfgang / Sager, Sven F. (Hrsg.) (2001): Text- und Gesprächslinguistik / Linguistics of Text and Conversation. 2. Halbband. Berlin / New York. S. 658-675.

Ehlich, Konrad (1994): Funktionen und Struktur schriftlicher Kommunikation. In: Schrift und Schriftlichkeit. Ein interdisziplinäres Handbuch internationaler Forschung 1. Hrsg. von Hartmut Günther und Otto Ludwig (Handbücher zur Sprach- und Kommunikationswissenschaft 10/1). Berlin. S. 18-41.

Engberg, Jan / Nølke, Henning (1997): Die Verwendung der Negation nicht in deutschen Landgerichtsurteilen. In: Engberg, Jan / Trosborg, Anna (Hrsg.): Linguists and Lawyers - Issues we confront. Arbeiten zu Sprache und Recht. Tostedt. S. 161-189.

Fix, Ulla (2008): Texte und Textsorten: sprachliche, kommunikative und kulturelle Phänomene. Berlin.

Gloning, Thomas (2010): Funktionale Textbausteine in der historischen Textlinguistik. Eine Schnittstelle zwischen der Handlungsstruktur und der syntaktischen Organisation von Texten. In: Ziegler, Arne (Hrsg.) (2010): Historische Textgrammatik und Historische Syntax des Deutschen: Traditionen, Innovationen, Perspektiven. Berlin / New York. S. 173-194.

Greule, Albrecht (2012a): Sakralität. Studien zu Sprachkultur und religiöser Sprache. Herausgegeben von Sandra Reimann und Paul Rössler. Tübingen. 
Greule, Albrecht (2012b): Textsorten der Kanzleisprachen. In: Greule, Albrecht / Meier, Jörg / Ziegler, Arne (Hrsg.) (2012): Kanzleisprachenforschung. Ein internationales Handbuch. Berlin / Boston. S. 283-286.

Greule, Albrecht / Meier, Jörg / Ziegler, Arne (Hrsg.) (2012): Kanzleisprachenforschung. Ein internationales Handbuch. Berlin / Boston.

Heinemann, Wolfgang (2001a): Aspekte der Textsortendifferenzierung. In: Brinker, Klaus / Antos, Gerd / Heinemann, Wolfgang / Sager, Sven F. (Hrsg.): Text- und Gesprächslinguistik. Discourse and Conversation. Ein internationales Handbuch zeitgenössischer Forschung. Berlin / New York. S. 523-546.

Heinemann, Wolfgang (2001b): Textsorte - Textmuster - Texttyp. In: Brinker, Klaus / Antos, Gerd / Heinemann, Wolfgang / Sager, Sven F. (Hrsg.): Text- und Gesprächslinguistik. Discourse and Conversation. Ein internationales Handbuch zeitgenössischer Forschung. Berlin / New York. S. 507-523.

Janich, Nina (2008): Textlinguistik: 15 Einführungen. Tübingen.

Kuhn, Hugo (1969): Versuch einer Literaturtypologie des deutschen 14. Jahrhunderts. In: Typologia litterarum. Fs. M. Wehrli. Zürich. S. 261-280.

Lerchner, Gotthard (2002): Schriften zum Stil. Vorträge zur Ehrung Gotthard Lerchners anlässlich seines 65. Geburtstages und Aufsätze des Jubilars. Leipzig.

Meier, Jörg (2004): Städtische Kommunikation in der Frühen Neuzeit. Frankfurt am Main.

Meier, Jörg / Ziegler, Arne (2001): Stadtbücher als Textallianzen. Eine textlinguistische Untersuchung zu einem wenig beachteten Forschungsgegenstand. In: Schwarz, Alexander / Abplanalp Luscher, Laure (Hrsg.) (2001): Textallianzen am Schnittpunkt der germanistischen Disziplinen. Bern u.a. S. 217-245.

Meier, Jörg / Ziegler, Arne (2004): Textsorten und Textallianzen in städtischen Kanzleien. In: Simmler, Franz (Hrsg.) (2004): Textsortentypologie und Textallianzen von der Mitte des 15. bis zur Mitte des 16. Jahrhunderts. Akten zum Internationalen Kongress in Berlin. 21. bis 25. Mai 2003 (Berliner Sprachwissenschaftliche Studien 6). Berlin. S. 129-166.

Messina, Chiara (2013): Höchstrichterliche Urteile: Varietätenspezifische Textbausteine (AT-CH-D). In: Brambilla, Marina / Gerdes, Joachim / Messina, Chiara (Hrsg.): Diatopische Variation in der deutschen Rechtssprache. Berlin. S. 53-92.

Mihm, Arend (1999): Funktionen der Schriftlichkeit in der städtischen Gesetzgebung des Spätmittelalters. In: Zeitschrift für germanistische Linguistik. Deutsche Sprache in Gegenwart und Geschichte 27/1 (1999). S. 13-37.

Rachoinig, Sigrid (2009): Wir tun kund und lassen dich wissen. Briefe, Urkunden und Akten als spätmittelalterliche Grundformen schriftlicher Kommunikation, dargestellt anhand der Lebenszeugnisse Oswalds von Wolkenstein. Frankfurt am Main.

Schmidt-Wiegand, Ruth (1996): Gebrauchssituationen im Spiegel der Mitüberlieferung. Die deutschen Rechtsbücher des 13. und 14. Jahrhunderts in ihren Codices. In: Meier, Christel / Hüpper, Dagmar / Keller, Hagen (Hrsg.): Der Codex im Gebrauch. Akten des Internationalen Kolloquiums 11.-13. Juni 1992 (Münstersche Mittelalter-Schriften 70). München. S. 69-86.

Schwarz, Alexander (2001): Vorwort. In: Schwarz, Alexander / Abplanalp Luscher, Laure (Hrsg.) (2001): Textallianzen am Schnittpunkt der germanistischen Disziplinen. Bern u.a. S. 9-15.

Simmler, Franz (1996): Teil und Ganzes in Texten. Zum Verhältnis von Textexemplar, Textteilen, Teiltexten, Textauszügen und Makrostrukturen. In: Daphnis 25 (1996). S. 597-625.

Simmler, Franz (2001): Gesamtsatzstrukturen, ihre Ermittlungsverfahren und Textfunktionen in Luthers Übersetzungen des Matthäus-Evangeliums 1522 und 1545. In: Meier, Jörg / Ziegler, Arne (Hrsg.): Deutsche Sprache in Europa. Geschichte und Gegenwart. Festschrift für Ilpo Tapani Piirainen zum 60. Geburtstag. Wien. S. 139-153. 
Simmler, Franz (2007): Liturgische Textsorten und Textallianzen. In: Wissenschaften im Kontakt. Kooperationsfelder der Deutschen Wissenschaft. Festschrift für Albrecht Greule zum 65. Geburtstag. Hrsg. von Sandra Reimann und Katja Kessel. Tübingen. S. 451-468.

Steger, Hugo / Deutrich, Karl-Helge / Schank, Gerd / Schütz, Eva (Hrsg.) (1974): Redekonstellation, Redekonstellationstyp, Textexemplar, Textsorte im Rahmen eines Sprachverhaltensmodells. Begründung einer Forschungshypothese. Jahrbuch 1972 des Instituts für deutsche Sprache. S. 39-97.

Vogel, Kathrin (2012): Corporate Style: Stil und Identität in der Unternehmenskommunikation. Wiesbaden.

Waligóra, Krystyna (2009): Die Dekrete des Krakauer Oberhofs (1456-1504) als Textallianzen und Textsorten. Kraków.

Wiktorowicz, Józef (2007): Die Textsorten und Textallianzen in der deutschen Kanzleisprache von Krakau. In: Studien zu Textsorten und Textallianzen um 1500. Hrsg. von Jörg Meier und Ilpo Tapani Piirainen (Germanistische Arbeiten zur Sprachgeschichte 5). Berlin. S. 275-286.

Wiktorowicz, Józef (2011): Krakauer Kanzleisprache. Forschungsperspektiven und Analysemethoden. Warszawa.

Wolf, Norbert Richard (2009): Historische Textsorten und Stil / Historical varieties and style. In: Fix, Ulla / Gardt, Andreas / Knape, Joachim (Hrsg.) (2009): Rhetoric and Stylistics. Ein internationales Handbuch historischer und systematischer Forschung / An International Handbook of Historical and Systematic Research. Band 2 / Volume 2 (HSK 31.2). Berlin. S. 1691-1705.

Ziegler, Arne (2003): Städtische Kommunikationspraxis im Spätmittelalter. Historische Soziopragmatik und Historische Textlinguistik (Germanistische Arbeiten zur Sprachgeschichte 2). Berlin.

Ziegler, Arne (2005): Syntaktische Prinzipien älterer deutscher Fachtexte. Überlegungen zu pragmatischen Aspekten einer mittelhochdeutschen Prosasyntax. In: Syntax. Althochdeutsch-Mittelhochdeutsch. Eine Gegenüberstellung von Metrik und Prosa. Akten zum Internationalen Kongress an der Freien Universität Berlin, 26.-29. Mai 2004. Hrsg. von Franz Simmler unter Mitarbeit von Claudia Wich-Reif und Yvon Desportes (Berliner Sprachwissenschaftliche Studien 7). Berlin. S. 271-292.

Ziegler, Arne (Hrsg.) (2010): Historische Textgrammatik und Historische Syntax des Deutschen: Traditionen, Innovationen, Perspektiven. Berlin/New York.

\section{Abstracts}

Die in den niederschlesischen Archiven aufbewahrten Stadtbücher bilden in ihrer Gesamtmenge auf den ersten Blick eine mehr oder weniger geschlossene Textsorte. Wenn man sie aber texttypologisch und textlinguistisch im Einzelnen untersucht, stellt es sich in vielen Fällen heraus, dass sie viel heterogener sind als es zu sein schiene. Ein durchschnittliches Stadtbuch ist nämlich oftmals ein thematisch und formal recht uneinheitliches Konstrukt. Vor allem in den kleineren Stadtkanzleien waren die Rats- oder Gerichtsbücher sehr mannigfaltig, denn man verzeichnete in ein und demselben Buch ganz unterschiedliche Angelegenheiten oder Informationstypen, für die die größten Städte getrennte Bücher führten. So sind viele Stadtbücher Ansammlungen von Texten verschiedener Sorten, wobei neben kalligraphisch gestalteten Urkunden von kollektivem Belang flüchtig aufgezeichnete Notizen individueller Geltung auftauchen, die für die Geschichte des Ortes keine größere Bedeutung hatten. Aus diesem Grunde lässt sich das ,Stadtbuch“ eher mit dem Begriff, Textallianz' bezeichnen. Im vorliegenden Beitrag wird versucht, diese Annahme an einem ausgewählten Textbeispiel nachzuweisen.

Schlüsselwörter: Stadtbuch, Kanzleisprache, Mittelhochdeutsch, Frühneuhochdeutsch, Handschriften, Niederschlesisch, Textallianz, deutsche Sprachgeschichte 


\section{The manuscript of the First City Book from Schweidnitz in light of text-alliances and text-types problematic}

The city books preserved in the Lower Silesian archives at first sight form in their total amount more or less one closed type of text. But when they are examined in detail from the text-typological and text-linguistic perspective, it turns out in many cases that they are much more diverse than they seem to be. An average city book is often thematically and formally a rather mixed construct. Especially in the offices of smaller towns, where the books of councils or courts were varied, you could find in the same book quite different matters or types of information, for which the largest cities kept many separate books. So there are many city books that are, so to speak, collections of different types of texts, where in addition calligraphically designed certificates of collective concern and fleetingly recorded individual application notes emerge, which had no greater significance for the history of the place. For this reason, it is possible to call the city books rather "text-alliances" than only a "texttype". In this paper an attempt is made to prove this assumption based on a selected example of a medieval text from Lower-Silesia.

Keywords: town-books, chancellor-languages, Middle-High-German, Early-New-High-German, manuscripts, Lower-Silesian language, text-alliances, the history of the German language

Marek Biszczanik

Uniwersytet Zielonogórski

Instytut Filologii Germańskiej

Al. Wojska Polskiego 71A

65-001 Zielona Góra

Polen

E-Mail: marek-biszczanik@wp.pl 